\title{
Low 25-OH vitamin D levels at time of diagnosis and recurrence of ovarian cancer
}

\author{
Teresa Granato $^{1} \cdot$ Lucia Manganaro ${ }^{2} \cdot$ Luca Petri $^{3} \cdot$ Maria Grazia Porpora $^{4}$. \\ Valentina Viggiani ${ }^{3}$ - Antonio Angeloni ${ }^{3} \cdot$ Emanuela Anastasi $^{3}$
}

Received: 28 July 2015 / Accepted: 2 September 2015 / Published online: 9 September 2015

(C) International Society of Oncology and BioMarkers (ISOBM) 2015

\begin{abstract}
The objective of this study was to evaluate the correlation between $25-\mathrm{OH}$ vitamin $\mathrm{D}$ and ovarian cancer as a diagnostic marker or recurrence disease marker. We studied the following: (1) 61 women without gynecologic diseases, (2) 45 women affected by benign ovarian disease, (3) 46 women with recent diagnosis of ovarian cancer, (4) 26 follow-up women with recurrent ovarian cancer, and (5) 32 follow-up women with stable ovarian cancer. The $25-\mathrm{OH}$ vitamin D was quantified with LUMIPULSE ${ }^{\circledR} \mathrm{G} 25-\mathrm{OH}$ vitamin D on LUMIPULSE ${ }^{\circledR}$ G 1200 (Fujirebio, Japan). As a threshold value, identified by ROC curve analysis, $20.2 \mathrm{ng} /$ $\mathrm{mL}$ (sensitivity $73.3 \%$, specificity $84 \%$ ) was chosen corresponding to the limit between sufficient and insufficient 25$\mathrm{OH}$ vitamin D according to the WHO. Low $25-\mathrm{OH}$ vitamin D levels were observed in $26 \%$ of women without gynecologic diseases, in $80 \%$ of women with recent diagnosis of ovarian cancer and in $24 \%$ women affected by benign ovarian diseases $(p<0.001)$. The follow-up study showed an insufficient level of $25-\mathrm{OH}$ vitamin D in $73 \%$ women with recurrent ovarian cancer and in $47 \%$ women with stable ovarian cancer
\end{abstract}

Emanuela Anastasi

emanuela.anastasi@uniroma1.it

1 CNR-IBPM, National Research Council, Viale Regina Elena 324, 00161 Rome, Italy

2 Department of Radiological, Oncological, and Pathological Sciences, "Sapienza" University of Rome, Rome, Italy

3 Department of Molecular Medicine, "Sapienza" University of Rome, Rome, Italy

4 Department of Gynecology, Obstetrics and Urology, "Sapienza" University of Rome, Rome, Italy $(p<0.0003)$. This study showed that patients with ovarian cancer are often insufficient in 25-OH vitamin D compared to women with benign ovarian diseases. The women with recurrent ovarian cancer presented more often low levels compared to women with stable ovarian cancer. This study suggests that $25-\mathrm{OH}$ vitamin $\mathrm{D}$, due to its antiproliferative properties, can be a good marker for ovarian cancer also.

Keywords 25-OH vitamin D · Biomarker · Epithelial ovarian cancer $\cdot$ Follow-up

\section{Introduction}

Ovarian cancer is a devastating disease with a high reversion rate. Due to a typically asymptomatic early stage and lack of early diagnostic tools, the disease is usually diagnosed in a late stage. Currently, a combination of physical examination and serum biomarker measurement are the pillars of the diagnosis [1].

A lot of different factors such as obesity, smoke, and diet with a high content of starch and/or fat, can be associated to the increase of ovarian carcinoma risk [2]. Meanwhile, a slight decrease in risk seems to be associated with a diet rich of fibers; carotene; vitamins C, E, and D; and unsaturated fatty acids $[3,4]$.

Vitamin D is a group of five different vitamins: vitamins D1, D2, D3, D4, and D5. Vitamin D is a steroid-like hormone which can be obtained from two sources: (1) exposure to ultraviolet B radiation from sunlight, that converts 7-dehydrocholesterol to vitamin D3 in the skin and (2) assimilation of vitamin D (D2 or D3) in diet and supplements [5-7]. The two most important forms with similar biological activity are vitamin D2 (ergocalciferol) and vitamin D3 (cholecalciferol). 
The 25-OH vitamin $\mathrm{D}$ (the sum of vitamins $\mathrm{D} 2$ and $\mathrm{D} 3$ ) has an important metabolic function in a lot of tissues and systems but, recently, its involvement in onset and progression of many diseases including cancer is becoming increasingly evident [8-10]. In addition to mineral homeostasis, vitamin D regulates gene expression in a lot of cellular processes, such as apoptosis, proliferation, differentiation, and a series of immuno-modulator processes that are directly or indirectly associated with cancer. In the last decade, the interest of many researches is focused on the relationship between vitamin D3 and carcinogenesis [11].

Recent studies showed that vitamin D3 levels influence cancer development, in particular, that VDR Fok1 polymorphism correlated with increased risks for ovarian cancer [12].

It is already known that some cancers are more frequent in regions where the sun exposure is lower, with a (more than $50 \%$ ) increased risk of breast, colon, prostate cancer (and partially, pancreas and ovarian cancer plus some lymphomas) in subjects with low vitamin D levels [10]. More recent data indicate that vitamin $\mathrm{D}$ supplementation can reduce the cancer incidence [8]. The results of these studies are not always concordant and some in vitro studies could not be confirmed in vivo. The co-occurrence of tumors or tumor markers and low vitamin $\mathrm{D}$ is now the subject of many scientific research. In this study, we evaluated the correlation between vitamin D3 levels and ovarian cancer using baseline (time of first diagnosis) serum samples of patients with high levels of CA125 and HE4 (in comparison to their respective cutoff values).

\section{Patients and methods}

\section{Patients}

All subjects included in the follow-up study were patients referred to the Oncologic Unit A, of the Policlinico Umberto I, Rome, Italy, from January 2012 to December 2014.

Group 1 consists of 61 normal blood donors (median age 57 years, range 21-65) with clinical and ultrasound (US) outcome negative for ovarian masses.

Group 2 consists of 45 patients with benign ovarian tumors (median age 62 years, range 20-74) with clinical and instrumental diagnosis of benign adnexal disease. Mean diameter of the cysts was $57 \pm 30.6 \mathrm{~mm}$ (range 20-110).

Group 3 consists of 46 women with newly diagnosed ovarian cancer (median age 68 years, range 28-91). Clinical and histological diagnosis was defined according to the International Federation of Gynecology and Obstetrics (new revised FIGO, 2014) staging [13]. Epithelial ovarian cancer (EOC) histology and grading of the EOC was in accordance with the current classification and guidelines $[14,15]$. Clinical and histopathological characteristics of the 46 patients are summarized in Table 1.

Group 4 consists of 26 follow-up women with recurrent ovarian cancer (median age 56 years, range 40-65).

Group 5 consists of 32 follow-up women with stable ovarian cancer (median age 59 years, range 35-68).

All patients signed written informed consent to participate in the study. At enrolment, medical history was collected and peripheral blood samples were drawn and immediately sent to the laboratory for analysis of 25-OH vitamin D.

\section{Sample collection}

Patient sera were acquired following a standard collection protocol. Samples were collected in a Red Top Vacutainer, clotted $60-90 \mathrm{~min}$ and centrifuged for $10 \mathrm{~min}$ at $1300 \times \mathrm{g}$. The serum fractions were aliquoted and stored at $-80{ }^{\circ} \mathrm{C}$ until analysis.

\section{Evaluation of EOC relapse}

Recurrent EOC, unlike other solid tumors, tends to present without accompanying symptoms and forms multiple small implantations, particularly in the small intestine and mesentery, which cannot be readily detected using conventional imaging techniques $[16,17]$. Computed tomography (CT) has low sensitivity for detecting disease recurrence, probably due to its inability to detect small peritoneal implants and normalsized lymph node metastases [18, 19]. The following criteria for recurrence of the disease (peritoneal carcinomatosis and lymph node dissemination) were established in the follow-up study

Table 1 Clinic pathological characteristics of the newly diagnosed ovarian cancer patients $(n=46)$

\begin{tabular}{llll}
\hline Disease status & & Number & Percent (\%) \\
\hline Histology & Serous & 24 & 52.17 \\
& Mucinous & 6 & 13.0 \\
& Undifferentiated & 13 & 28.26 \\
\multirow{3}{*}{ Grade } & Others & 3 & 6.52 \\
& 1 & 1 & 2.17 \\
& 2 & 20 & 43.47 \\
Stage & 3 & 25 & 54.34 \\
& I & 6 & 13.0 \\
& II & 5 & 10.86 \\
& III & 6 & 13.00 \\
& IV & 29 & 63.0 \\
\hline
\end{tabular}


(1) Peritoneal carcinomatosis. Single or multiple sites of micro-nodular implants $(<1 \mathrm{~cm})$ above or below the mesocolon; diffuse macro-nodular implants $(>1.5 \mathrm{~cm})$ on the bowel surface or mesenterial implants; and omental involvement with marked thickening (omental cake).

(2) Lymph node dissemination. The onset of lymph node $>1 \mathrm{~cm}$ and/or the $20 \%$ size increase of pre-existing lymph nodes. Stable ovarian cancer was diagnosed when the disease did not follow any of the parameters described above.

\section{Measurement of 25-OH vitamin D}

25-OH vitamin D levels were measured with the LUMIPULSE $^{\circledR}$ G 1200 (Fujirebio, Japan), an assay system that allows quantitative measurements and is based on chemiluminescent enzyme immunoassay (CLEIA) technology. The LUMIPULSE $^{\circledR} G 25-\mathrm{OH}$ vitamin D uses a novel two-step sandwich method [20] for detecting $25-\mathrm{OH}$ vitamin $\mathrm{D}$. The assay uses a solid-phase and alkaline phosphatase (ALP)-labeled monoclonal antibodies that specifically bind to the analyte forming antigen-antibody immunocomplexes. ALPlabeled monoclonal antibody binds to the antigen of the immunocomplexes, and adamantyl-1,2-dioxetane phosphate (AMPPD) contained in the substrate solution is dephosphorylated by the catalysis of ALP indirectly conjugated to the particles. A luminescent signal is generated by the cleavage reaction of dephosphorylated AMPPD and reflects the amount of antigen in the sample.

\section{Statistical analysis}

The statistical significance of difference between the number of patients affected by ovarian cancer and that of patients affected by other benign pathologies showing values of the 25-OH deficiency was assessed using a chi-square test for categorical variables. In terms of the diagnostic accuracy of the assays, the performance was assessed by estimation of receiver operating characteristic (ROC) curve for ovarian cancer cases versus benign gynecologic diseases. The area under the ROC curve (AUC) was calculated by MedCalc V 4.30 Software.

\section{Results}

\section{5-OH vitamin D normal levels in our healthy population}

Sixty blood donors were included in this study. These samples were obtained through a collaboration with the Blood Bank of the Policlinico Umberto I. As a threshold value, identified by ROC curve analysis, $20.2 \mathrm{ng} / \mathrm{mL}$ (sensitivity $73.3 \%$, specificity $84 \%$ ) was found to correspond to the limit between sufficient and insufficient $25-\mathrm{OH}$ vitamin D according to the WHO (Fig. 1).

\section{5-OH vitamin D levels in ovarian cancer}

We evaluated 25-OH vitamin D values in 61 blood donors (group 1), 45 patients with benign ovarian diseases (group 2 ), and 46 patients with recent diagnosis of ovarian cancer (group 3). The patients were divided according to their 25$\mathrm{OH}$ vitamin D status into a deficient $(<20 \mathrm{ng} / \mathrm{mL})$ and a nondeficient $(>20 \mathrm{mg} / \mathrm{mL}$ ) group. Insufficient levels of $25-\mathrm{OH}$ vitamin D were observed in 16/61 (26\%) group 1 women, in 11/45 (24\%) Group 2 patients, and 34/46 (80\%) group 3 patients. We observed a statistical difference $(p$ value $<0.001)$ between the values observed in ovarian cancer patients (group 3 ) versus benign gynecology diseases (group 2) patients and healthy donors (group 1) (Fig. 2).

We also investigated the correlation of $25-\mathrm{OH}$ vitamin D concentrations with disease recurrence. We evaluated $25-\mathrm{OH}$ vitamin D levels in 58 patients divided in two groups of women affected by recurrent disease (group 4, $n=26$ ) and women with stable disease (group 5, $n=32$ ). The follow-up study showed an insufficient level of 25-OH vitamin D in 19/26 (73\%) women with recurrent ovarian cancer (group 4) and in 16/32 (47\%) women with stable ovarian cancer (group 5) $(p<0.0003)$ (Fig. 3).

\section{Discussion}

Ovarian cancer, as a notorious "silent killer," is one of the most lethal gynecological neoplasms in women living in

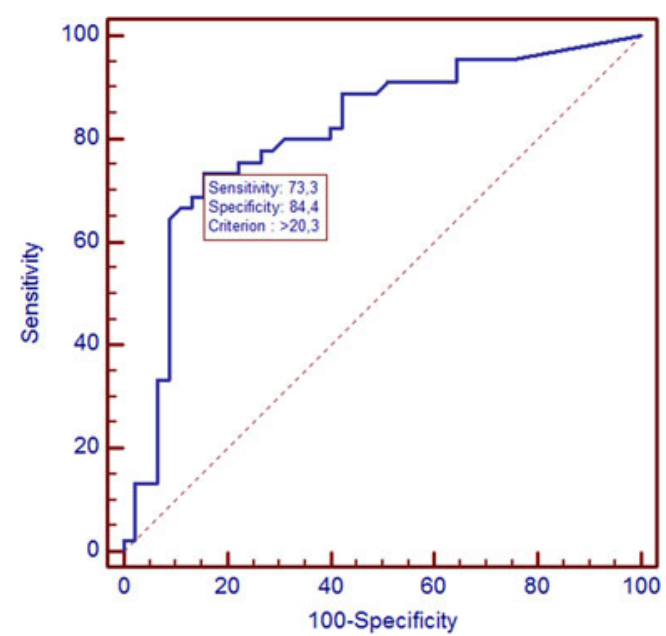

Fig. 1 ROC curve analysis for determining 25-OH Vitamin D insufficiency. The threshold value was determined to be $20.2 \mathrm{ng} / \mathrm{mL}$ (sensitivity $73.3 \%$, specificity $84 \%$ ) with an area under the ROC curve 0.811 (95\% CI $0.71-0.88)$ 


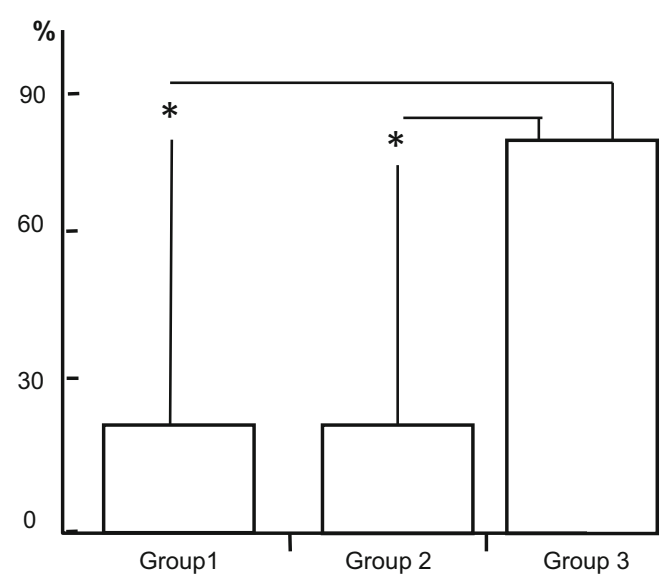

Fig. 2 Percentage of individuals affected by $25-\mathrm{OH}$ vitamin D insufficiency in healthy women (group 1), patients with benign gynecology diseases (group 2), and patients affected by ovarian cancer (group $3 ;{ }^{*} p<0.001$ )

industrialized countries [21]. At present, the global annual incidence is approximately 204,499 cases per year with a mortality of 124,860 patients per year [22]. The expected number of new ovarian cancer cases in Europe in 2012 was 65,538 with 42,704 deaths [1]. Due to the development of surgery and chemotherapy with empirically optimized combinations of conventional agents, survival rate of ovarian cancer currently approximates $30 \%$ [23]. If carcinoma is diagnosed at an early stage, it has an excellent prognosis since it can be treated before spreading to surrounding tissue [24].

Currently, as many as $70 \%$ of patients are diagnosed at International Federation of Gynecology and Obstetrics (FIGO) stages III and IV. This late diagnosis is generally related to the asymptomatic behavior of the disease in the early stages. Although certain EOC screening tests have been shown to decrease mortality rates, the possibility of efficient

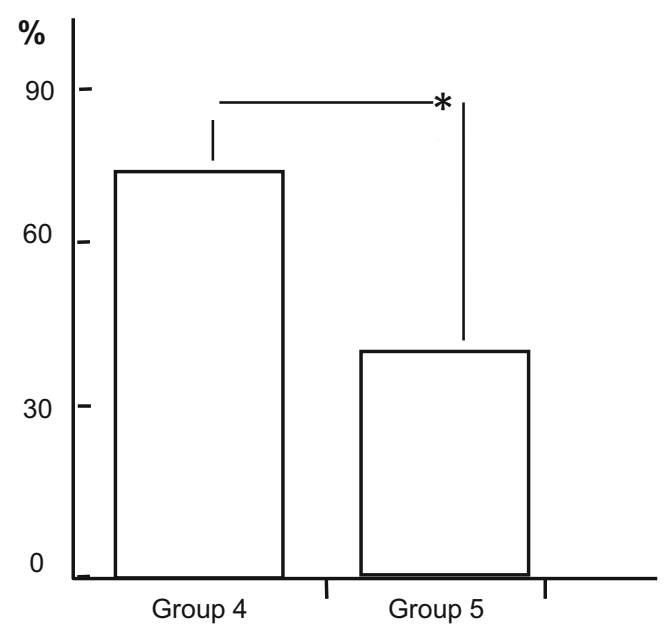

Fig. 3 Percentages of individuals with $25-\mathrm{OH}$ vitamin D insufficiency at follow-up in the group of patients with recurrence (group 4) and stable disease (group $5 ;{ }^{*} p<0.0003$ ) screening that may be used in normal practice remains elusive $[25,26]$.

Recently, vitamin $\mathrm{D}$ has been found to be less expressed in some cancers (breast, lung) [10]. Moreover, this decrease in cancer could help discriminate between benign and malignant diseases [27]. The present study confirmed the findings from other cancers and demonstrated that $25-\mathrm{OH}$ vitamin $\mathrm{D}$ is decreased at time of diagnosis in nearly all patients affected by ovarian cancer and that only a small percentage of patients affected by gynecological benign diseases had levels below the cutoff for $25-\mathrm{OH}$ vitamin $\mathrm{D}[10,28]$. These results could be interpreted according to the $25-\mathrm{OH}$ vitamin $\mathrm{D}$ antiproliferative effects and its ability to block cell mitosis in the G1 phase [29].

In addition to the search of specific markers for the determination of the early stages of any cancer form, it is just as important to find markers capable of following the remission from disease as response to therapy and in the event of the unfortunate recurrence of the disease.

At present, a multimodal approach including the utilization of tumor biomarkers associated with ovarian cancer, besides imaging techniques, seems to be the best strategy in the assessment of tumor spread, recurrence, and/or the response to treatment. CT and PET-CT play an important role in early detection of recurrence ovarian cancer, even if there is no agreement on which type of follow-up examination should be used or when it should be employed. However, the value of PET-CT is limited in areas of physiologic radiotracer accumulation and in small implants size. Diffusion-weighted MRI imaging may be advantages for overcoming dissemination. The combined interpretation of diffusion-weighted images with conventional MRI has been shown to increase accuracy in the staging of ovarian cancer and in the follow-up [30].

Our study shows a higher percentage of vitamin D deficiency in patients affected by disease recurrence in respect to the group with stable disease, suggesting that vitamin D deficiency could be a risk factor for disease recurrence. To this aim, the synergy of vitamin $\mathrm{D}$ with other vitamins should be studied to determine its relevance in the natural immunity and blocking of angiogenesis. Recent studies with animal models suggested potent anti-metastatic activities of $25-\mathrm{OH}$ vitamin D [31].

A broad scientific bibliography has established, enhanced, and confirmed the preventive and therapeutic activity of 25$\mathrm{OH}$ vitamin $\mathrm{D}$ in neoplastic diseases. Recently, cellular receptors (VDR) of 25-OH vitamin D have been identified and proven to have anti-neoplastic effects [12]. A lot of studies on synthetic, new-generation vitamin $\mathrm{D}$ are ongoing. They are focused on reducing the calcemic activity that can produce, if in excess, serious and irreversible damages and, meanwhile, increase the anti-neoplastic rule in synergy with retinoid for cellular differentiation activities. In addition to the in vitro and animal model studies, clinical and epidemiologic studies also 
confirmed the anti-carcinogenic properties of $25-\mathrm{OH}$ vitamin $\mathrm{D}$ and its derivatives in tumoral prevention and therapy.

In conclusion, this study has shown that vitamin D may be able to provide a valuable contribution both to the diagnosis of ovarian cancer and to controlling its recurrence. Thus, the search for biomarkers which (1) can predict as early as possible the occurrence of ovarian or other gynecological cancers and (2) can foresee, in spite of treatment, the recurrence of these diseases, can help in taking the fundamental steps needed to control and, ultimately, find a definitive cure for these diseases.

Acknowledgments This study is founded by the University of Rome Sapienza. The authors are thankful to Giuseppina Gennarini, Barbara Colaprisca, and Silvestra Tudini for their excellent technical assistance.

\section{Conflicts of interest None}

\section{References}

1. Ledermann JA, Raja FA, Fotopoulou C, Gonzalez-Martin A, Colombo N, ESMO Guidelines Working Group, et al. Newly diagnosed and relapsed epithelial ovarian carcinoma: ESMO clinical practice guidelines for diagnosis, treatment and follow-up. Ann Oncol. 2013;24:24-32.

2. Li K, Hüsing A, Fortner RT, Tjønneland A, Hansen L, Dossus L, et al. An epidemiologic risk prediction model for ovarian cancer in Europe: the EPIC study. Br J Cancer. 2015;5. doi:10.1038/bjc. 2015.22.

3. di Masi A, Leboffe L, De Marinis E, Pagano F, Cicconi L, Rochette-Egly C, et al. Retinoic acid receptors: from molecular mechanisms to cancer therapy. Mol Aspects Med. 2015;41C:1115.

4. Xie J, Poole EM, Terry KL, Fung TT, Rosner BA, Willett WC, et al. A prospective cohort study of dietary indices and incidence of epithelial ovarian cancer. J Ovarian Res. 2014;7(1):112-4.

5. Wang G, Zhang Q, Xu N, Xu K, Wang J, He W, et al. Associations between two polymorphisms (FokI and BsmI) of vitamin D receptor gene and type 1 diabetes mellitus in Asian population: a meta analysis. PLoS One. 2014;9(3):e89325.

6. Attar R, Gasparri ML, Donato VD, Yaylim I, Halim TA, Zaman F, et al. Ovarian cancer: interplay of vitamin D signaling and miRNA action. Asian Pac J Cancer Prev. 2014;15(8):3359-62.

7. Watanabe R, Inoue D. Current topics on vitamin D. Anti-cancer effects of vitamin D. Clin Calcium. 2015;25(3):373-80.

8. Wranicz J, Szostak-Wegierek D. Health outcomes of vitamin D. Part II. Role in prevention of diseases. Rocz Panstw Zakl Hig. 2014;65(4):273-9.

9. Gandini S, Gnagnarella P, Serrano D, Pasquali E, Raimondi S. Vitamin D receptor polymorphisms and cancer. Adv Exp Med Biol. 2014;810:69-105.

10. Shui IM, Mondul AM, Lindström S, Tsilidis KK, Travis RC, Gerke T,et al. Circulating vitamin D, vitamin D-related genetic variation, and risk of fatal prostate cancer in the National Cancer Institute Breast and Prostate Cancer Cohort Consortium. Cancer. 2015;2. doi:10.1002/cncr.29320.

11. Nemazannikova N, Antonas K, Dass CR. Vitamin D: metabolism, molecular mechanisms, and mutations to malignancies. Mol Carcinog. 2014;53(6):421-31.
12. Li S, Xu H, Li SC, Qi XQ, Sun WJ. Vitamin D receptor rs 2228570 polymorphism and susceptibly to ovarian cancer: a meta-analysis. Tumour Biol. 2014;35(2):1319-22.

13. Mutch DG, Prat J. 2014 FIGO staging for ovarian, fallopian tube and peritoneal cancer. Gynecol Oncol. 2014;133(3):401-4. doi:10. $1016 / \mathrm{j}$.

14. Bonfrer JM, Duffy MJ, Kulpa J, Rustin GJ, Soletormos G, Torre GC, et al. CA125 in ovarian cancer: European Group on Tumor Markers guidelines for clinical use. Int J Gynecol Cancer. 2005; 15: 679-91.

15. Aebi S, Castiglione M. Newly and relapsed epithelial ovarian carcinoma: ESMO clinical recommendations for diagnosis; treatment and follow-up. Ann Oncol. 2009;20(Supp4):iv21-3.

16. Tempany CM, Zou KH, Silverman SG, Brown DL, Kurtz AB, McNeil BJ. Staging of advanced ovarian cancer: comparison of imaging modalities - report from the Radiological Diagnostic Oncology Group. Radiology. 2000;215:761-7.

17. Manganaro L, Michienzi S, Vinci V, Falzarano R, Saldari M, Granato T, et al. Serum HE4 levels combined with CE CT imaging improve the management of monitoring women affected by epithelial ovarian cancer. Oncol Rep. 2013;30:2481-7.

18. Gu P, Pan LL, Wu SQ, Sun L, Huang G. CA 125, PET alone, PET$\mathrm{CT}, \mathrm{CT}$ and MRI in diagnosing recurrent ovarian carcinoma: a systematic review and meta-analysis. Eur J Radiol. 2009;71:164-74.

19. Midulla C, Manganaro L, Longo F, Viggiani V, Frati L, Granato T, et al. HE4 combined with MDCT imaging is a good marker in the evaluation of disease extension in advanced epithelial ovarian carcinoma. Tumour Biol. 2012;33:1291-8.

20. Falzarano R, Viggiani V, Michienzi S, Longo F, Tudini S, Frati L, et al. Evaluation of a CLEIA automated assay system for the detection of a panel of tumor markers. Tumour Biol. 2013;34(5):3093-100.

21. Ozols RF. Recurrent ovarian cancer: evidence-based treatment. J Clin Oncol. 2002;20:1161-3.

22. Colombo N, Peiretti M, Parma G, Lapresa M, Mancari R, Carinelli $\mathrm{S}$, et al. Newly diagnosed and relapsed epithelial ovarian carcinoma: ESMO clinical practice guidelines for diagnosis, treatment and follow-up. Ann Oncol. 2010;21:23-30.

23. Bast Jr RC, Hennessy B, Mills GB. The biology of ovarian cancer: new opportunities for translation. Nat Rev Cancer. 2009;9(6):41528.

24. Lynch HT, Drescher K, Knezetic J, Lanspa S. Genetics biomarkers, hereditary cancer syndrome diagnosis, heterogeneity and treatment: a review. Curr Treat Options Oncol. 2001;15:429-42.

25. Guideline No. 62RCOG/BSGE Joint Guideline Royal College guidelines 2011. Management of suspected ovarian masses in premenopausal women. Green-top.

26. Mutch D, Denny L, Quinn M. FIGO Committee on Gynecologic Oncology. Hereditary gynecologic cancers. Int J Gynaecol Obstet. 2014;124:189-92.

27. Granato T, Porpora MG, Longo F, Angeloni A, Lucia Manganaro L, Anastasi E. He4 in the differential diagnosis of ovarian masses. Clin Chim Acta. 2015;446:147-55.

28. Walentowicz-Sadłecka M, Sadłecki P, Walentowicz P, Grabiec M. The role of vitamin $\mathrm{D}$ in the carcinogenesis of breast and ovarian cancer. Ginekol Pol. 2013;84(4):305-8.

29. Irazoqui AP, Boland RL, Buitrago CG. Actions of 1,25(OH)2-vitamin D3 on the cellular cycle depend on VDR and p38 MAPK in skeletal muscle cells. J Mol Endocrinol. 2014;53(3):331-43.

30. Low RN, Sebrechts CP, Barone RM, Muller W. Diffusion-weighted MRI of peritoneal tumors: comparison with conventional MRI and surgical and histopathologic findings-a feasibility study. AJR Am J Roentgenol. 2009;193:461-70.

31. Irving AA, Plum LA, Blaser WJ, Ford MR, Weng C, Clipson L, et al. Cholecalciferol or 25-hydroxycholecalciferol neither prevents nor treats adenomas in a rat model of familial colon cancer. J Nutr. 2015;145(2):291-8. 